\title{
Modelos de percolação elétrica aplicados para compósitos poliméricos condutores
}

\section{Percolation electrical models applied to conductive polymers composites}

\author{
Paulo Henrique da Silva Leite Coelho ${ }^{1 *}$ e Ana Rita Morales ${ }^{2}$ \\ 'Departamento de Tecnologia Química, Centro de Ciências Exatas e Tecnologias, \\ Universidade Federal do Maranhão - UFMA, São Luís, MA, Brasil \\ ${ }^{2}$ Departamento de Engenharia de Materiais e Bioprocessos, Faculdade de Engenharia Química, \\ Universidade Estadual de Campinas - UNICAMP, Campinas, SP, Brasil \\ *piyke.coelho@gmail.com
}

\begin{abstract}
Resumo
O presente artigo apresenta a aplicação e adequação dos modelos de percolação elétrica em trabalhos experimentais e teóricos da literatura para compósitos poliméricos condutores. Foi realizado um levantamento das publicações referentes aos modelos estudados para os diferentes tipos de cargas condutoras mais aplicadas na preparação destes compósitos, tais como pós metálicos, grafite, negro de fumo, nanofibras e nanotubos de carbono. A discussão está apresentada quanto à adequação dos modelos ao comportamento dos compósitos na influência das cargas nas propriedades elétricas de matrizes poliméricas.
\end{abstract}

Palavras-chave: compósitos poliméricos, condutividade elétrica, modelos de percolação.

\begin{abstract}
This paper presents the application and adjustment of electrical percolation models in conductive polymer composites. Different models have been proposed for different types of conductive fillers applied in composites preparation, such as metal powders, graphite, black carbon, carbon nanotubes and nanofibers. The discussion was carried out considering the consistency of the model on the behavior of these fillers and their influence on the electrical properties of polymer matrices.
\end{abstract}

Keywords: polymer composites, electrical conductivity, percolation models.

\section{Introdução}

A incorporação de partículas eletricamente condutoras em uma matriz isolante polimérica é um exemplo importante da modificação de materiais em áreas até então restrita aos metais, tais como, piezo elétricos ${ }^{[1]}$, adesivos $\operatorname{condutivos}^{[2]}$, roupas e artigos antiestáticos ${ }^{[3]}$, blindagem eletromagnética ${ }^{[4]}$ e sensores químicos ${ }^{[5]}$. A esta classe de materiais é dada a denominação de compósitos condutores poliméricos que apresentam muitas vantagens em comparação aos metais, como por exemplo, resistência à corrosão, menor densidade, processabilidade e custo ${ }^{[6]}$.

A adição de cargas eletricamente condutoras, tais como, partículas metálicas ${ }^{[7-9]}$, nanofolhas de grafite ${ }^{[10-12]}$, negro de fumo (NF) ${ }^{[13,14]}$ e nanotubos de carbono (NTC ${ }^{[15-18]} \mathrm{em}$ uma resina polimérica isolante tem sido o principal recurso para a obtenção de compósitos condutores. Entre estas cargas destacam-se os NTC por seu potencial na obtenção de sistemas nanoestruturados altamente condutores com excelentes propriedades mecânicas ${ }^{[17,18]}$.
A principal questão sobre os nanocompósitos poliméricos condutores está relacionada com a variação da condutividade em função da concentração das partículas condutoras, onde se observa existir uma concentração crítica a partir da qual ocorre um aumento na condutividade do sistema. Este fenômeno pode ser explicado pela teoria da percolação.

Vários modelos matemáticos foram desenvolvidos para descrever o fenômeno de percolação e uma revisão abrangente foi feita por Lux $1993^{[19]}$, considerando fatores estruturais micrométricos dos sistemas. Desde então, estes modelos continuam a ser aplicados e discutidos. Mais recentemente, destaca-se a importância de novos materiais obtidos a partir de cargas cujas dimensões são nanométricas.

Bauhofer e Kovacs ${ }^{[20]}$ apresentaram uma revisão em 2009 sobre a percolação elétrica destes compósitos poliméricos, evidenciando dados de mais de uma centena de artigos relacionados à determinação do limiar de percolação. Foram considerados diversos parâmetros relacionados à síntese, tratamento, dimensionalidade, bem como métodos de 
preparação e dispersão das cargas na determinação dos limiares de percolação. Aspectos importantes como a aplicação da lei das potências, valores do expoente crítico e a condutividade máxima obtida para os compósitos pela variação desses parâmetros foram discutidos. Este artigo, embora bastante abrangente, limitou-se ao estudo da condutividade elétrica em sistemas com NTC, não sendo discutida a aplicação dos modelos de percolação para estes sistemas.

Diante deste quadro o presente artigo tem como objetivo ampliar esta discussão a partir dos trabalhos relatados na literatura relativos à aplicação dos modelos de percolação elétrica para compósitos poliméricos obtidos com cargas condutoras nanométricas.

\section{Compósitos Poliméricos}

Compósitos de poliméricos com cargas à base de carbono recebem, recentemente, uma atenção considerável, tanto na pesquisa acadêmica quanto industrial, devido à possibilidade em se combinar alta condutividade elétrica com baixo peso específico e facilidade de processamento ${ }^{[21,22]}$.

Existem basicamente quatro tipos de cargas de carbono: negro de fumo (NF), grafite $(\mathrm{G})$, fibras de carbono (FC) e nanotubos de carbono (NTC), sendo que cada tipo apresenta distintas características na formação de compósitos condutores, sendo que nos últimos anos, um dos sistemas mais promissores são os nanocompósitos preparados com NTC em matrizes poliméricas ${ }^{[23,24]}$.

Desde a descoberta dos NTC ${ }^{[25]}$ grande atividade acadêmica tem sido dedicada à exploração de suas propriedades mecânicas, térmicas e elétricas ${ }^{[15,16]}$, pela preparação de materiais nanocompósitos que se beneficiam de uma ou mais destas características, na busca de aplicações para diversos tipos de dispositivos ${ }^{[17]}$. Logo, os NTC têm grande potencial para uma variedade de aplicações como termoplásticos condutores elétricos ou térmicos, adesivos condutivos, suporte de catalisadores, aplicações biológicas, filtração de ar e água, entre outras ${ }^{[18]}$.

A utilização dos NF é um dos recursos mais comuns para modificação de polímeros com a finalidade de conferir condutividade elétrica ou propriedades antiestáticas. $\mathrm{O}$ avanço tecnológico na dispersão de cargas, e o desenvolvimento de NF de alta estrutura revelou uma melhora significativa em compósitos poliméricos com NF. Onde antes uma concentração relativamente elevada de carga, cerca de 10 vol.\% ${ }^{[26,27]}$, seria necessária para atingir a percolação elétrica gerando um aumento na viscosidade do compósito e perda nas propriedades mecânicas, na última década são relatadas concentrações que chegam próximas a $1 \%$ dependendo da morfologia, tamanho e da dispersão das cargas condutoras utilizadas ${ }^{[28-31]}$. As propriedades elétricas de compósitos NF/polímeros têm sido estudadas ${ }^{[13,14]}$, mas não há um número vasto de artigos concentrados em avaliar quantitativamente a condutividade elétrica, bem como a aplicação de modelos que explicam o fenômeno da percolação para cargas particuladas, como o NF.

Comparando-se estas duas principais opções, NF e NTC, os NTC despertam um maior interesse devido à sua alta condutividade, até $10.000 \mathrm{~S} / \mathrm{cm}^{[32]}$ e sua alta razão de aspecto, normalmente $>1000$, o que facilita a obtenção de um baixo limiar de percolação elétrica ${ }^{[33,34]}$, sendo relatadas concentrações críticas abaixo de $0,1 \%$ em massa ${ }^{[17]}$.

Park et al. ${ }^{[35]}$ estudaram compósitos de PMMA e NTC e observaram um aumento de até 12 ordens de grandeza na condutividade elétrica do compósito em relação ao PMMA puro. Zhao et al. ${ }^{[36]}$ também analisaram a condutividade, mas de compósitos de NF e NTC com polipropileno (PP), e observaram aumento da condutividade na ordem de grandeza de 10 e 11, respectivamente para NF/PP e NTC/PP.

Assim como os NTC as nanofolhas de grafite (NFG) são materiais promissores na preparação de compósitos poliméricos, devido às altas razão de aspecto, condutividade elétrica e condutividade térmica. Recentemente tem havido um interesse crescente em materiais compósitos de polímeros/NFG ${ }^{[10-12]}$, pois estes apresentam baixo limiar de percolação elétrico, abaixo de $1 \%$ em volume ${ }^{[37,38]}$, e a modelagem deste comportamento tem sido feita com base no alinhamento e na orientação das partículas e na sua influência nas propriedades destes compósitos ${ }^{[39-41]}$.

A combinação de dois tipos de cargas, uma particulada e outra na forma de fibras ou nanotubos, vem sendo estudada $^{[30,34,42-45]}$ e apresenta-se promissora na formação das redes condutoras. As cargas condutoras com alta razão de aspecto (FC e NTC) atuam como transportadoras de cargas elétricas e as partículas de menor razão de aspecto (G e NF) têm a função de preencher os espaços promovendo a interligação entre as fibras ou os nanotubos no compósito polimérico $^{[46,47]}$.

Estudos mostram que cargas com diferentes formas incorporadas aos materiais poliméricos resultam em um limiar de percolação com concentrações menores quando comparados aos compósitos com apenas um tipo de partícula ${ }^{[20,42,48-50]}$. Sumfleth et al. ${ }^{[50]}$ relataram que, devido ao efeito sinérgico na formação de rede e no transporte de carga elétrica, a mistura de NTC e NF em matriz epóxi levou a um comportamento elétrico similar quando comparado aos nanocompósitos com apenas NTC. Além disso, a adição de NF aumenta a condutividade máxima alcançada com o aumento da concentração de cargas.

Para sistemas baseados em compósitos de borracha de estireno-butadieno, Bokobza et al. ${ }^{\left[{ }^{[1]}\right.}$ encontraram uma melhor dispersão dos NTC, menores valores de resistividade elétrica e redução no limiar de percolação, passando de 10 pcr para valores entre 2 e 3 pcr, quando se utilizou NTC e NF juntos.

Em matrizes de termoplásticos, vários resultados são relatados na literatura sobre o uso de dois tipos de cargas de carbono $^{[30,34,42,44,45,52,53]}$. A mistura de FC e NF em polietileno foi descrito por Calleja et al. ${ }^{[52]}$, que relataram que as FC forneceram o transporte de cargas elétricas em grandes distâncias e as partículas de NF melhoraram os contatos entre as fibras levando a um aumento de condutividade elétrica. Lee et al. ${ }^{[53]}$ observaram uma diminuição da resistividade elétrica ao adicionar NTC em compósitos contendo NF, no entanto os autores não discutiram os efeitos sinérgicos entre as duas cargas. Socher et al. ${ }^{[34]}$ analisaram o limiar de percolação elétrico de compósitos de poliamida 12 com NTC e NF, e concluíram que, apesar da influência do teor de cada carga na concentração crítica de percolação nenhum efeito sinergético entre as cargas foi encontrado para estes sistemas. 
Compósitos poliméricos com partículas metálicas condutoras são também de interesse para muitos campos da engenharia ${ }^{[-9]}$. Este interesse é resultante das características elétricas destes compósitos, próximas à dos metais, enquanto que as propriedades mecânicas e os métodos de processamento são típicos dos polímeros ${ }^{[54]}$. Observou-se experimentalmente, que a condutividade elétrica de compósitos metal-polímero depende predominantemente de características como a forma e o tamanho das partículas, concentração volumétrica e o arranjo espacial das cargas na matriz ${ }^{[55-57]}$.

\section{Preparação e Dispersão de Compósitos Poliméricos Condutores}

Diversos métodos de preparação de compósitos condutores baseados em matrizes termoplásticas ou termofixas têm sido relatos na literatura ${ }^{[30,34,45,58-60]}$. Dentre estes métodos, alguns são mais usualmente citados como: mistura em solução, polimerização in situ e mistura no estado fundido. Embora existam diferentes métodos, todos afetam as propriedades finais do compósito, sendo que as nanocargas devem estar bem dispersas e distribuídas na matriz polimérica. A distribuição está associada à homogeneidade da amostra enquanto que a dispersão descreve o estado de aglomeração ${ }^{[61]}$.

A dispersão dos NTC é um problema que difere bastante da dispersão de outras cargas convencionais, como as partículas esféricas, fibras de carbono e silicatos em camada. Isto porque os NTC possuem uma alta razão de aspecto $(>1000)$ com diâmetro na escala nanométrica e área superfícial extremamente alta. Além disso, os NTC comerciais são fornecidos na forma de feixes fortemente entrelaçados, resultando em uma dificuldade inerente na dispersão ${ }^{[18]}$.

Dentre os métodos de preparação e dispersão de compósitos poliméricos condutores citados acima, os mais utilizados e eficientes são apresentados a seguir.

\subsection{Solução}

Este é o método mais comum para a fabricação de nanocompósitos, pois é favorável à dispersão nanométrica das partículas. A mistura em solução envolve três etapas principais: dispersão das cargas em solvente adequado à solubilização do polímero; mistura desta solução com polímero (em temperatura ambiente ou temperatura elevada) e recuperação do compósito pela evaporação do solvente, processo também conhecido por casting ${ }^{[59]}$. A dispersão das cargas no solvente é a etapa fundamental para a obtenção de um compósito com ótimas propriedades. A técnica de ultra-sonificação é bastante utilizada para a dispersão de nanopartículas em meios líquidos. Consiste basicamente na aplicação de uma energia ultrassônica para a agitação das partículas na solução. $\mathrm{O}$ choque das ondas promove a desarticulação dos aglomerados e a separação das partículas. Esta técnica é geralmente utilizada em laboratórios onde se utiliza um banho de ultra-som ou uma sonda ultra-sônica, conhecida como sonicador. $\mathrm{O}$ tratamento de pré dispersão por ultra-som pode ser muito agressivo, e devido à facilidade de danificação das cargas, principalmente os NTC, o tempo e a potência devem ser bem estabelecidos quando este equipamento for empregado ${ }^{[18]}$.

\subsection{Polimerização in situ}

Esta técnica consiste na preparação e dispersão das partículas condutoras em monômero seguido por polimerização. Tal como acontece com a mistura em solução, as cargas podem ser previamente dispersas em líquido (monômero ou solvente) e, posteriormente todo o sistema é submetido ao processo de polimerização. Uma das vantagens deste processo é a possibilidade da incorporação de uma alta concentração de cargas dispersas devido à forte interação destas com a matriz promovida pela polimerizaçãa ${ }^{[59,61]}$.

A técnica de polimerização in situ em conjunto com a dispersão ultra-sônica foi citada por Park et al.$^{[35]}$ na preparação de nanocompósitos de NTC e polimetacrilato de metila com o objetivo de estudar as propriedades elétricas e reológicas dos compósitos formados.

\section{Teoria da Percolação}

Para a discussão teórica de mecanismos de condução em compósitos poliméricos, necessita-se de um bom entendimento sobre a condução de corrente elétrica entre partículas incorporadas na matriz. Alguns fatores, como a aproximação dos contatos entre partículas, geração e condução de calor entre a matriz e as cargas são determinados pelas propriedades destes materais ${ }^{[62]}$. No entanto, neste trabalho, o objetivo não é discutir a fundo a modelagem destes mecanismos de condução, e sim revisar modelos que simulem a concentração crítica de cargas para tornar o compósito condutor.

A condutividade elétrica de compósitos formados por uma matriz polimérica isolante e uma partícula condutora depende criticamente da concentração de carga condutora adicionada à matriz. Baixas frações levam a uma grande distância média entre as partículas, limitando o sistema à condutividade da matriz, que tipicamente apresenta valores na casa de $10^{-15} \mathrm{~S} / \mathrm{cm}^{[2]}$. Quando uma quantidade de carga suficiente é adicionada ao polímero, a aproximação das partículas permite a condução, momento no qual se observa um intervalo de concentração para o qual a condutividade pode mudar drasticamente em várias ordens de grandeza para pequenas variações no teor de carga. A este comportamento dá-se o nome de limiar de percolação elétrica. Para maiores concentrações, o número de caminhos condutores aumenta até a formação de uma rede tridimensional. Nessa faixa a condutividade é alta e torna-se menos sensível às pequenas alterações na concentração volumétrica da carga na matriz ${ }^{[62]}$.

A condutividade elétrica em materiais compósitos pode ser explicada pela teoria de percolação. A teoria da percolação foi matematicamente desenvolvida em 1957 por Broabent e Hammersley ${ }^{\left[{ }^{[3]}\right]}$ com o objetivo de descrever fenômenos físicos aleatórios como a percolação de fluidos em meios porosos, propagação de incêndios em florestas e fluxo de corrente elétrica através de materiais, por meio de análises estatísticas.

Desde o trabalho pioneiro, modelos de percolação atraem o interesse do mundo acadêmico, em especial de matemáticos, físicos, químicos, biólogos e engenheiros. Trata-se de uma classe de modelos que descrevem as propriedades de um determinado sistema desordenado. Existem basicamente 
dois tipos de modelos de percolação: a percolação por sítios e a percolação por ligação ${ }^{[62]}$.

No primeiro, considera-se uma rede quadrada bidimensional formada por $S$ sítios vazios (Figura 1), na qual todos os sítios da rede são separados com a mesma distância entre seus vizinhos mais próximos, de tal forma que, em cada local da rede há uma probabilidade $p$ de um sítio estar preenchido (Figura $1 b$ ) e probabilidade $1-p$, deste sítio estar vazio (Figura 1a). No segundo modelo, as ligações são estabelecidas entre os dois sítios vizinhos preenchidos em uma rede (Figura 1c). Cada ligação tem uma probabilidade $p$ de existir e a probabilidade $1-p$ de não conectar os sítios vizinhos. Em ambos os casos, as estruturas de pontos conectados podem ser definidos como aglomerados de forma que é possível a formação de um caminho de condução. Neste caso, o valor de $p$ para o qual atinge-se esta condição pode ser chamado de probabilidade crítica $\left(p_{c}\right)$, ou limiar de percolação, que será o valor determinante para que haja a percolação em um sistema ${ }^{[19]}$.

Para compósitos poliméricos condutores a percolação ocorrerá quando as partículas condutoras estiverem suficientemente bem dispersas e conectadas para que haja a formação de um caminho ininterrupto pela rede dimensional proporcionando fluxo de corrente. Esta concentração é representada no modelo como o número de partículas devidamente dispersas, como sítios, ou posições preenchidas, e é chamada de concentração crítica (ou limiar de percolação), $\varphi_{C}$. Quando a concentração de sítios $\varphi$ se aproxima da concentração crítica $\varphi_{C}$, surge o primeiro caminho ininterrupto de sítios ocupados de um eletrodo ao outro, podendo-se dizer que o sistema percolou.

Então, pode-se definir que para $\varphi<\varphi_{C}$ o sistema não conduz; para $\varphi>\varphi_{C}$ o sistema tem uma condutividade alta e, para $\varphi=\varphi_{C}$ o sistema percola, sendo esta condição que permite uma primeira avaliação na alteração significativa da condutividade. $\mathrm{O}$ modelo de percolação por ligação segue o mesmo raciocínio anterior, entretanto, para esse modelo, os sítios da rede quadrada bidimensional são conectados por ligações. Num sistema real, os sítios ou as ligações correspondem às partículas ou aglomerados de partículas que ocupam espaços na rede (sítios) ou que se conectam (ligações - Figura 1c).

\section{Modelos de Percolação para Compósitos Poliméricos de Partículas Condutoras e Matriz Polimérica Isolante}

Uma revisão abrangente sobre a aplicação dos modelos para explicar o comportamento da condutividade elétrica em materiais compostos de matriz isolante e partícula condutora foi feita por $\mathrm{Lux}^{[19]} \mathrm{em} 1993$. Os modelos foram classificados como modelos estatísticos, termodinâmicos, geométricos e de estruturas orientadas. No entanto, alguns destes modelos descrevem apenas em escala micrométrica a formação da rede percolativa nas misturas.

Os dois modelos precursores desenvolvidos foram os modelos de Bruggeman ${ }^{[64]}$ e de Garland e Tanner ${ }^{[65]}$. Com base nestes, outros autores desenvolveram estudos para os quais foram incluídos parâmetros relevantes anteriormente não considerados, como orientação, forma e fração volumétrica, que influenciam diretamente na condutividade nestes compósitos $^{[66-69]}$.

Mais recentemente, a tecnologia se desenvolveu na utilização de cargas com dimensões nanométricas e a adequação destes modelos vem sendo discutida considerando-se estruturas geométricas onde a forma, distribuição e orientação das partículas condutoras passam a serem fatores determinantes ${ }^{[30,31,34,36,44,61]}$.

\subsection{Modelo clássico estatístico de percolação}

Modelos estatísticos de percolação sobre o fenômeno da percolação elétrica para materiais formados com partículas condutoras em matrizes isolantes são citados na literatura e tiveram seu pioneirismo nos estudos de Kirkpatrick $^{[63]}$ e Zallen $^{[64]}$

A base do modelo clássico considera o desenvolvimento de redes em matrizes regulares finitas. Por meio de simulação computacional é possível determinar a concentração de sítios ou ligações existentes de acordo com a lei das potências, comumente usada em fenômenos de transição de fases, dada por:

$$
\sigma \propto\left(p-p_{c}\right)^{\beta}
$$

onde $\beta$ é o expoente crítico e $p$ e $p_{C}$ variam de acordo com a propriedade analisada.
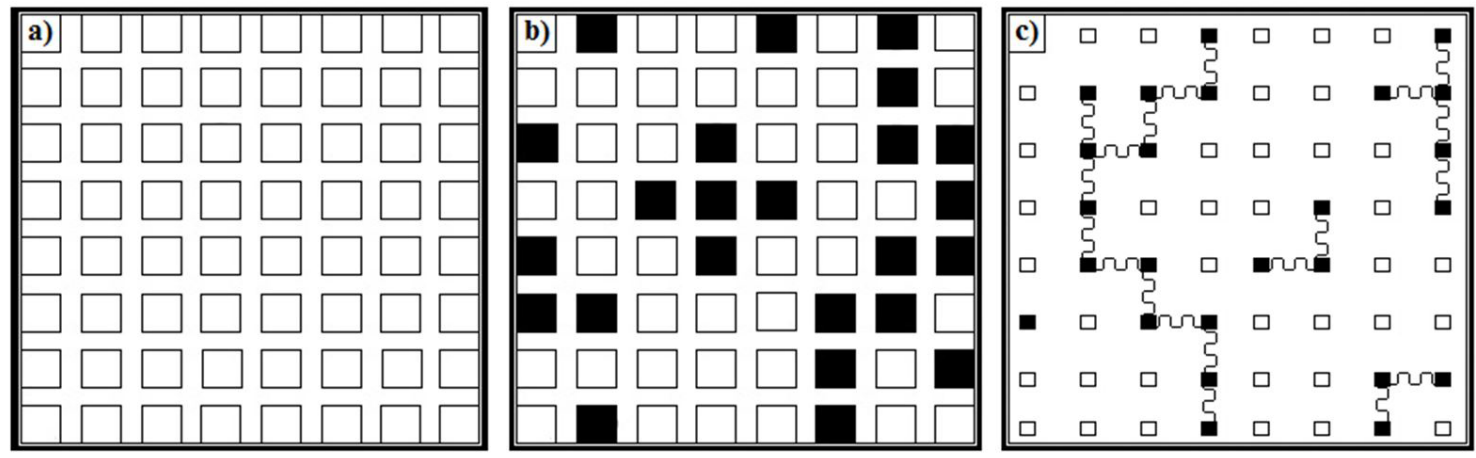

Figura 1. Exemplo de uma rede bidimensional (a) de sítios desocupados (1-p) e (b) de percolação por sítios ocupados ( $p$ ) e (c) de percolação por ligação. 
Considerando as propriedades elétricas específicas dos materiais, a equação, correlaciona a condutividade da mistura com a fração volumétrica da partícula condutora e assume a seguinte relação:

$$
\sigma=\sigma_{0}\left(\varphi-\varphi_{c}\right)^{\beta} \operatorname{com} \varphi_{\mathrm{c}}=p_{\mathrm{c}}^{\text {sitio }} . \mathrm{V}
$$

onde $\sigma$ é a condutividade da mistura, $\sigma_{O}$ a condutividade da partícula condutora, $\varphi$ a fração volumétrica da partícula, $\varphi_{C}$ é a fração volumétrica crítica de percolação, $\beta$ é o parâmetro que determina o aumento da condutividade acima de $\varphi_{C}$ e $v$ é o fator relacionado à localização de uma partícula em um ponto específico da rede. Os valores de $\varphi_{C}$ e $\beta$ dependem apenas da dimensão da rede. Kirkpatrick ${ }^{[70]}$ apresentou os seguintes valores para o expoente $\beta$ : para modelo de percolação por ligação $\beta=1,6 \pm 0,1$ e para o modelo de percolação por sítio $\beta=1,5 \pm 0,1$.

A Tabela 1 apresenta os valores de fração volumétrica crítica de Zallen ${ }^{[71]}$ para diferentes dimensionalidades. A partir da Equação 2 e dos dados da Tabela 1 estudos desenvolvidos com misturas de polímero com $\mathrm{NF}^{[28]}$ e de polímero com $\mathrm{FC}^{[72]}$ mostraram adequação dos resultados experimentais com os teóricos. Porém, este modelo de Kirkpatrick ${ }^{[70]}$ não considera aspectos de forma e dimensões das partículas condutoras. Autores mostraram em seus estudos que a concentração de percolação depende da forma das partículas condutoras, e que quanto mais irregular a partícula, menor será a concentração crítica necessária para a percolação ${ }^{[73]}$.

Estudos têm demonstrado que o modelo estatístico é adequado para os resultados experimentais de $\mathrm{NTC}^{[74]}, \mathrm{NF}^{[75]}$ e partículas metálicas ${ }^{[76]} \mathrm{em}$ matriz isolante.

Janzen $^{[77]}$ desenvolveu um modelo de percolação partindo do conceito do número médio de contatos entre as partículas condutoras. Ele determinou o número 1,5 como o número médio de contatos entre as partículas, tomando como base os resultados de Kirkpatrick ${ }^{[70]}$, e propôs a seguinte equação:

$$
\varphi_{c}=\frac{1}{1+0,67 z \rho \varepsilon}
$$

onde $\varphi_{C}$ é o limiar de percolação, $z$ é o número de coordenação (número de vizinhos mais próximos) em uma rede específica, $\rho$ é a densidade das partículas condutoras e $\varepsilon$ é o volume específico dos poros destas partículas. Isto descreveu adequadamente o sistema de compósitos sinterizados de NF em matriz polimérica.

\subsection{Modelo geométrico de percolação}

O modelo geométrico foi proposto por Slupkowski ${ }^{[78]}$ para explicar o fenômeno de percolação em sistemas de materiais isolantes e condutores em pó pré-misturados

Tabela 1. Concentração de percolação para diferentes dimensionalidades ${ }^{[19]}$.

\begin{tabular}{cclll}
\hline Dimensionalidade & $\begin{array}{c}\text { Estrutura da } \\
\text { Rede }\end{array}$ & $\boldsymbol{p}_{\mathbf{c}}^{\text {sítio }}$ & $\boldsymbol{p}_{\mathbf{c}}^{\text {ligação }}$ & $\boldsymbol{\varphi}_{\boldsymbol{c}}$ \\
\hline 2 & Triangular & 0,5 & 0,347 & 0,45 \\
2 & Quadrada & 0,593 & 0,5 & 0,47 \\
3 & Cubo simples & 0,312 & 0,249 & 0,163 \\
3 & Diamante & 0,428 & 0,388 & 1,146 \\
\hline
\end{tabular}

a seco e posteriormente sinterizados. O modelo assume que, durante o processo de sinterização, as partículas condutoras são dispostas de maneira regular na superfície das partículas isolantes. O autor assumiu o diâmetro das partículas isolantes e condutoras como o principal fator de influência no limiar de percolação. $\mathrm{O}$ arranjo pode ser assim descrito: as partículas condutoras formam uma camada de espessura $g$ na superfície das partículas isolantes de forma esférica, onde a corrente elétrica flui nesta superfície através dos pontos de contato. De acordo com os pressupostos de Slupkowski, estas considerações resultaram na Equação 4.

$$
\sigma=2 \pi \sigma_{c} \frac{d([x]+p)}{D \ln \left(1+\frac{D}{([x]+1) d}\right)}
$$

onde $\sigma$ é a condutividade elétrica do sistema, $\sigma_{C}$ é a condutividade elétrica da partícula condutora, $D$ e $d$ são os diâmetros das partículas isolantes e condutoras, respectivamente, $[x]$ é o valor inteiro de $x$, que representa o número de camadas completas de partículas condutoras em torno de cada partícula isolante, e $p$ é a probabilidade de formação de uma rede condutora de percolação na camada exterior. A relação dos diâmetros pode ser descrita utizando-se o modelo de duas dimensões de rede proposto por Scarisbrick ${ }^{[79]}$, conforme a Equação 5.

$$
[x]=\left[\left(\frac{1}{1-V_{f}}\right)^{1 / 3}-1\right] \frac{D}{2 d}
$$

com $V_{F}$ sendo a fração volumétrica das partículas.

Malliaris e Turner ${ }^{[80]}$, no seu estudo sobre a influência do tamanho de partícula na resistividade elétrica de compósitos de pó métalico em matriz polimérica, propuseram um modelo geométrico teórico partindo do pressuposto da formação de cadeias infinitas de pó metálico, a partir do modelo clássico estatístico porém, levando em consideração o raio das partículas da matriz e da carga condutora e propuseram a Equação 6.

$$
V_{A}=50 p_{c}\left[1+\left(\frac{\varnothing}{4}\right)\left(\frac{R_{p}}{R_{f}}\right)\right]^{-1}
$$

onde $p_{C}$ é a probabilidade, diferente de zero, para que caminhos infinitos formados por sítios adjacentes da rede ocupados por elementos condutores (por exemplo, $p_{C}=1 / 3$ para rede hexagonais, $1 / 2$ para redes quadradas e $2 / 3$ para redes triangulares), $\phi$ é um fator que depende no modo de ocupação das cargas condutoras (por exemplo, $\phi=1,11$ para hexagonal), $R_{P}$ e $R_{F}$ são o raio da partícula do polímero e da partícula condutora, respectivamente. Eles assumiram que as partículas de polímero são maiores que as cargas condutoras e que suas superfícies ficam uniformemente cobertas pelas cargas condutoras.

Young ${ }^{[81]}$ desenvolveu um estudo que evidencia uma relação de proporcionalidade entre o tamanho e a forma das partículas dos compósitos e o limiar de percolação. O sistema foi composto de NF disperso em politetrafluoretileno, ambos na forma de pó. Os resultados experimentais encontrados 
foram comparados com um conjunto de modelos de percolação geométricos e concluiu-se que estes modelos podem ser utilizados semi-quantitativamente para determinar baixos limiares de percolação elétrica. Estes modelos têm assumido que as partículas isolantes são esféricas ou cúbicas e que a determinação da área superficial destas partículas é essencial para a exatidão quantitativa de qualquer dos modelos de percolação geométrica. Apesar disto, o autor obteve uma melhor adequação ao assumir o formato elipsoidal das partículas condutoras e a distribuição de tamanho das partículas.

\subsection{Modelo do volume excluído}

O conceito de volume excluído tem sido proposto para estimar o limiar de percolação de compósitos contendo partículas não esféricas estocasticamente dispersas.

Balberg et al. ${ }^{[26]}$ basearam-se no conceito de volume excluído para propor seu modelo para os NTC. O volume excluído de um objeto pode ser definido como o volume em torno deste objeto onde o centro de outro objeto semelhante não pode penetrar ${ }^{[24,26]}$. Para uma esfera esta definição pode ser ilustrada pela Figura 2. Este conceito tem sido usado para a predição do limiar de percolação de cargas como NF, G, NTC onde estes objetos são considerados como esfera, disco e esferocilindro, respectivamente. Esta aplicação está voltada para sistemas nos quais os objetos possuem grande razão de aspecto, como é o caso dos nanotubos de carbono e considera que não há interação entre a carga e a matriz polimérica ${ }^{[26]}$.

Para Balberg et al. ${ }^{[26]}$ o limiar de percolação, $\varphi_{C}$, é inversamente proporcional ao volume excluído do objeto estudado, $V_{E X}$, conforme a relação descrita pela Equação 7.

$$
\varphi_{\mathrm{c}} \propto \frac{1}{V_{\mathrm{ex}}}
$$

De acordo com este modelo, para um sistema em 3D pode-se definir a fração volumétrica crítica de percolação das partículas através do volume total excluído das partículas $V_{E X}$ conforme a Equação ${ }^{[82]}$.

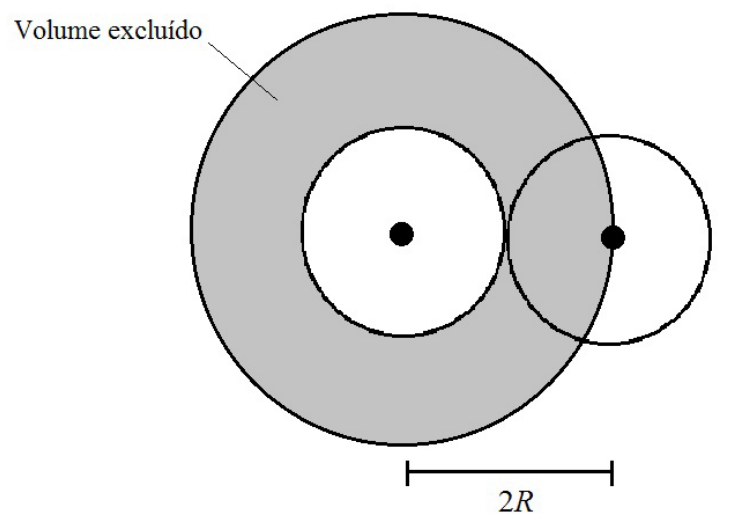

Figura 2. Representação do volume excluído de uma partícula esférica de raio $R$.

$$
\varphi_{\mathrm{c}}=1-\exp \left(\frac{\left\langle V_{\mathrm{ex}}\right\rangle V}{\left\langle V_{\mathrm{e}}\right\rangle}\right)=1-\exp \left(-N_{\mathrm{c}} V\right)
$$

onde $N_{C}$ é o número total de partículas, $V_{E}$ o volume excluído de cada partícula, e $V$ é o volume real da partícula.

Para esta forma de partícula com alta razão de aspecto definiu-se o objeto como um esferocilindro rígido (Figura 3) de comprimento $\mathrm{L}$ e raio $\mathrm{R}$ aleatoriamente orientados em $3 \mathrm{D}$, para o qual o volume excluído pode ser descrito pela Equação 9:

$$
V_{e x}=\frac{32 \pi}{3} R^{3}\left[1+\frac{3}{4}\left(\frac{L}{R}\right)+\frac{3}{8 \pi}\langle\operatorname{sen}(\theta)\rangle\left(\frac{L}{R}\right)^{2}\right]
$$

onde $<\operatorname{sen}(\theta)>$ é o valor médio de sen $(\theta)$, que descreve o alinhamento das partículas e $\theta$ é o ângulo entre as mesmas. Um valor de $\operatorname{sen}(\theta)=0$ significa que os esferocilindros estão perfeitamente alinhados. Para NTC aleatoriamente distribuídos o valor do ângulo é assumido como $\pi / 4$.

Bug et al. ${ }^{[83]}$ também propuseram um modelo para estudo da percolação elétrica para os NTC de comprimento $L$ e diâmetro $D$ definido pela Equação 11 .

$$
\left\langle V_{e}\right\rangle=\frac{4}{3} \pi D^{3}+2 \pi D^{2} L+2 D L^{2}
$$

O valor de $\left\langle V_{E}\right\rangle$ representa o volume excluído de cada partida e pode ser utilizado na equação Equação 8 para determinação da fração volumétrica crítica, como na Equação 10.

$$
\varphi_{\mathrm{c}}=1-\exp \left(\frac{\left\langle V_{\mathrm{ex}}\right\rangle\left[\frac{\pi}{4} D^{2} L+\frac{\pi}{6} D^{3}\right]}{\left[\frac{4 \pi}{3} D^{3}+2 \pi D^{2} L+2 D L^{2}\langle\operatorname{sen}(\theta)\rangle\right]}\right)
$$

Com base nas Equações 8, 9 e 10 outros autores ${ }^{[84,85]}$ desenvolveram estudos que tomaram como base o modelo do volume excluído ${ }^{[26,82]}$ e a adequação da geometria da Figura 3. Celzard et al. ${ }^{[84]}$ propuseram um modelo pela variação de diferentes ângulos de orientação das cargas, confirmando que para o valor de $\theta=\pi / 2$ e $<\operatorname{sen}(\theta)>\approx \pi / 4$, o menor da fração crítica mostrou-se $0,58<\varphi_{C}<1,15$. A avaliação da percolação definida para um comprimento $L$ e diâmetro $D$ transforma a Equação 11 na Equação 12.

$$
\varphi_{\mathrm{c}}=1-\exp \left(\frac{\left\langle V_{\mathrm{ex}}\right\rangle\left[\frac{\pi}{4} D^{2} L+\frac{\pi}{6} D^{3}\right]}{\left[\frac{4 \pi}{3} D^{3}+2 \pi D^{2} L+\frac{\pi}{2} D L^{2}\right]}\right)
$$

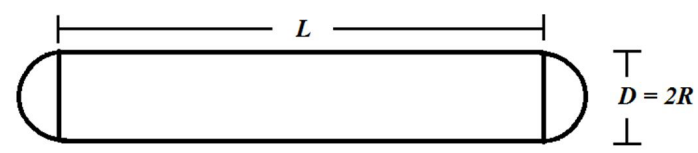

Figura 3. Estrutura do esferocilindro. 
Para cargas condutoras esféricas, como é o caso do NF, pode-se considerar que o volume excluído de cada esfera seja expresso pela Equação 13 e pode ser aplicado com o mesmo princípio utilizado nos NTC para se obter a concentração crítica de partículas para a formação da rede ${ }^{[86]}$.

$$
V_{\text {ex_esfera }}=\frac{32 \pi}{3} R^{3}
$$

Porém, as morfologias de compósitos de NF não são comumente encontradas como partículas esféricas, isto devido à difícil desagregação das partículas individuais dos aglomerados no processo de dispersão que acabam por se organizar em estruturas diversas e isto é um fator nem sempre considerado.

Deng et al. ${ }^{[87]}$ modelaram a percolação elétrica a partir da teoria do volume excluído para Polipropileno (PP) e NTC e de PP e aglomerados de NF, considerando o NTC como esferocilindros e as partículas de NF unidas como contas em um colar. Os autores observaram que os valores para o limiar de percolação para ambos, NTC e NF, foram coerentes para quando a orientação entre as cargas $(\theta)$ foi de $0-\pi / 6$, diferentemente da aleatoriedade de $\theta=\pi / 4$ do modelo de Balberg, Equação 9, tipicamente assumida em vários outros trabalhos ${ }^{[83,86,88]}$.

Estudos que tomam como base a teoria do volume excluído têm demonstrado, experimentalmente, a proporcionalidade citada na Equação $7^{[83,88]}$. A concentração volumétrica crítica encontrada pela Equação 8 ao ser aplicada à Equação 2, fundamentada pela lei das potências, permite a descrição do comportamento da condutividade das partículas condutoras em matrizes isolantes.

A grande maioria dos trabalhos teóricos descrevem modelos onde cargas condutoras são consideradas como retas ou bastões rígidos. No entanto, esta não é a realidade da estrutura dos NTC. A flexibilidade existente nestas cargas e a alta razão de aspecto formam um emaranhado de partículas na matriz polimérica. Isto dificulta a modelagem da estrutura e da orientação das partículas. Dalmas et al. ${ }^{[88]}$ mostraram que o limiar de percolação não é influenciado significativamente quando a razão de aspecto da carga é superior a 100. Já Berhan e Sastry ${ }^{[86,89]}$ apresentaram uma discussão mais ampla para sistemas com cargas com alta razão de aspecto, da ordem de centenas ou milhares. A discussão foi baseada na sobreposição de partículas, pois muitos trabalhos de modelagem consideram os NTC como cilindros permeáveis ou com "núcleo macio" (soft-core), que promove uma menor exigência computacional na simulação. Berhan e Sastry ${ }^{[86,89]}$ compararam esta situação com uma mais realista: a de que os NTC não têm superposição direta uns com os outros, sendo chamados de "núcleo duro" (hard-core), e que são ondulados e não bastões rígidos. Utilizando simulações de Monte Carlo foi demonstrado haver uma proporcionalidade inversa entre o limiar de percolação e o volume excluído para os dois modelos, "núcleo macio" e "núcleo duro", demonstrando também que a constante de proporcionalidade é governada pela razão de aspecto e que o modelo "núcleo macio" é mais apropriado para a modelagem de compósitos onde o mecanismo da condução elétrica é por tunelamento ${ }^{[86]}$. Se as cargas são altamente curvas ou mesmo enroladas, existe uma diferença significativa na modelagem em relação às cargas rígidas e retas, sendo que para cargas mais curtas o efeito da intensidade das curvas é muito mais pronunciado do que para as cargas longas, na predição do limiar de percolação ${ }^{[89]}$. Os autores observaram também que a escolha pelo tipo de núcleo que não considera a superposição das cargas leva a um erro que pode exceder $4 \%$ para os NTC com razão de aspecto menores de $400^{[89]}$.

Quanto ao limiar de percolação elétrica dos sistemas contendo dois tipos de cargas condutoras com diferentes formas, Sun et al. ${ }^{[42]}$ desenvolveram um estudo para NTC e NF baseado na percolação das cargas individuais com uma adaptação à teoria do volume excluído. O desenvolvimento deste estudo considera a Figura 3 e o modelo tem como base a Equação 7 proposta por Balberg ${ }^{[26]}$ e é descrito pela Equação 14.

$$
N_{c}=k \frac{V_{\text {unit }}}{V_{<e x>}}
$$

Os autores definem $V_{U N I T}$ como o volume unitário da carga condutora, $N_{C}$ como o número de objetos necessários em $V_{U N I T}$ para a percolação e, $k$ como a constante de proporcionalidade, que é obtida experimentalmente, e mostra a relação entre $N_{C} / V_{U N I T}$ e $1 / V_{<E X>}$, ou seja, a relação inversamente proporcional entre o número de objetos por unidade de volume de percolação em relação ao volume excluído do objeto, que está relacionado à forma e à disposição do objeto. A constante de proporcionalidade $k$ se torna unitária quando a razão de aspecto do objeto é significantemente grande e os objetos se encontram aleatoriamente orientados.

Se houver apenas um tipo de carga condutora, pode-se supor que a unidade de volume $V_{U N I T}$ é dividido por $N_{C}$ volumes menores equivalentes (chamados pequenos volumes), onde cada pequeno volume é $V_{<E X>} / k$. Quando uma partícula condutora existe em cada pequeno volume, a percolação ocorre e $N_{C} V_{<E X>} / k=V_{U N I T}$, o que equivale à Equação 14.

Portanto, se houver apenas NTC como carga, pode-se supor que a média de volume excluído de um objeto de NTC (individual ou aglomerado) é $V_{<N T C>}$ e a constante correspondente é $k_{N T C}$. Então, quando um objeto de NTC ocupa um pequeno volume, $V_{<N T C} / k_{N T C}$, a percolação ocorre (Figura 4a). Da mesma forma, se houver apenas NF como carga, tem-se a média de volume excluído de um objeto de $\mathrm{NF}$ (tamanho médio de agregados) como $V_{<N F\rangle}$ e a constante é $k_{N F}$. O pequeno volume é $V_{<N F} / K_{N F}$, e assim como para os NTC, quando um objeto de NF ocupa um pequeno volume, a percolação ocorre como mostrado na Figura $4 \mathrm{~b}$. Para todos os casos ocorre a percolação.

Para um sistema contendo os dois tipos de cargas de condutoras, NTC e NF, o volume unitário pode ser dividido em dois tipos de pequenos volumes, $V_{<N T C} / k_{N T C}$ e $V<_{N F}>/ K_{N F}$. Estes volumes estão ilustrados na Figura 4c, mas não representam a situação real do modelo para cada volume pequeno, mas sim uma situação aleatória encontrada nos compósitos formados pelas duas cargas. A Figura 4d ilustra os pequenos volumes de cada carga em uma situação extrema. Quando todos os pequenos volumes estão cheios, a percolação ocorre e pode ser descrita pela Equação 15. 
(a)

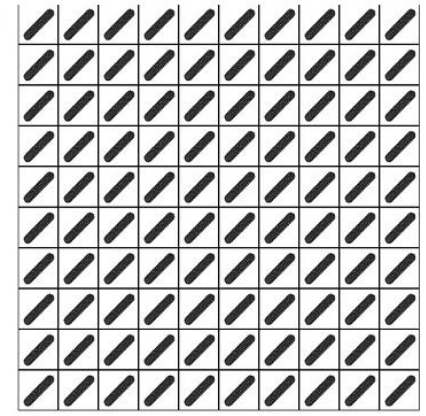

(c)



(b)

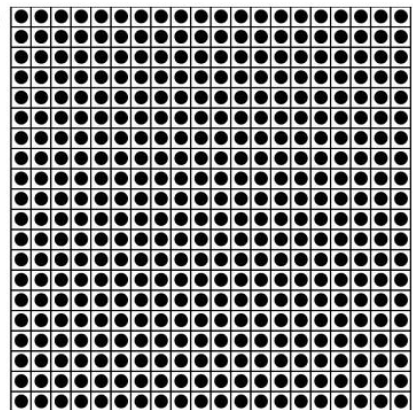

(d)

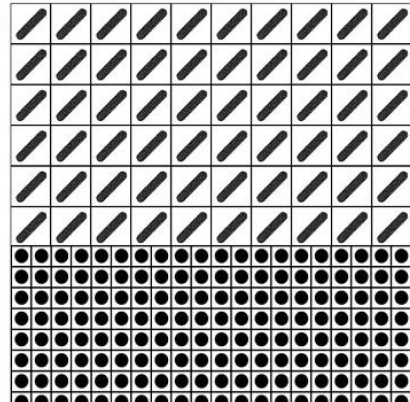

Figura 4. Ilustração do volume excluído para cargas simples: (a) NTC, (b) NF, (c) mistura real das cargas (NTC e NF) e (d) situação extrema de mistura. (Reproduzido com permissão de Sun et al. ${ }^{[90]}$ ).

$$
V_{u n i t}=N_{N T C}^{\prime} \frac{V_{<N T C>}}{k_{N T C}}+N_{N F}^{\prime} \frac{V_{<N F>}}{k_{N F}}
$$

onde $N^{\prime}{ }_{N T C}$ e $N^{\prime}{ }_{N F}$ são os números de objetos NTC e NF, respectivamente. Como o número de objetos de NTC (ou NF) é proporcional à fração volumétrica dos NTC (ou NF) em um determinado volume tem-se que:

$$
\begin{aligned}
& N_{N T C}^{\prime}=\frac{V_{N T C}}{\varphi_{c, N T C}} N_{N T C} \\
& N_{N F}^{\prime}=\frac{V_{N F}}{\varphi_{c, N F}} N_{N F}
\end{aligned}
$$

onde $V_{N T C}$ e $V_{N F}$ são as frações reais volumétricas dos objetos de NTC e NF, $\varphi_{C, N T C}$ e $\varphi_{C, N F}$ são as frações volumétricas de percolação das respectivas cargas se o volume unitário é preenchido pelas cargas separadas, e $N_{N T C}$ e $N_{N F}$, são os números de NTC e NF necessários para que ocorra a percolação.

Aplicando as Equações 16 e 17 na Equação 15 a Equação 18 pode ser obtida.

$$
V_{\text {unit }}=\frac{V_{N T C}}{\varphi_{c, N T C}} N_{N T C} \frac{V_{<N T C>}}{k_{N T C}}+\frac{V_{N F}}{\varphi_{c, N F}} N_{N F} \frac{V_{<N F>}}{k_{N F}}
$$

Da combinação das Equações 14 e 18:

$$
V_{u n i t}=\frac{V_{N T C}}{\varphi_{c, N T C}} V_{u n i t}+\frac{V_{N F}}{\varphi_{c, N F}} V_{\text {unit }}
$$

Como o $V_{U N I T}$ está presente em todos os termos, a Equação 19, torna-se:

$$
\frac{V_{N T C}}{\varphi_{c, N T C}}+\frac{V_{N F}}{\varphi_{c, N F}}=1
$$

Isto significa que quando $V_{N T C} / \varphi_{C, N T C}+V_{N F} / \varphi_{C, N F}=1$ as cargas condutoras começam a formar a rede percolada na matriz polimérica. Se $V_{N T C} / \varphi_{C, N T C}+V_{N F} / \varphi_{C, N F}>1$ as cargas estão em contato e a matriz se torna condutora. No caso oposto, $V_{N T C} / \varphi_{C, N T C}+V_{N F} / \varphi_{C, N F}<1$, as partículas estão separadas e a resistividade elétrica na matriz é alta. É importante notar que a Equação 20 é equivalente à média ponderada das frações de cada partícula condutora, e que a relação de igualdade exclui qualquer possibilidade de sinergismo. Portanto, a aplicação deste modelo permite comparar o valor da percolação teórica com o valor experimental e avaliar se este encontra-se abaixo do descrito pelo modelo. Se isto de fato acontecer, pode-se afimar que houve um sinergismo entre as cargas.

A fração volumétrica apresentada na Equação 20 pode ser generalizada para frações mássicas das partículas distintas $A$ e $B$. Visando a conveniência do uso prático e a necessidade de evitar a incerteza na determinação da densidade das partículas, a Equação 20 pode ser escrita na forma:

$$
\frac{m_{A}}{p_{c, A}}+\frac{m_{B}}{p_{c, B}}=1
$$

onde $m_{A}$ e $m_{B}$ são as massas das partículas condutoras e $p_{C, A}$ e $p_{C, B}$ são as frações mássicas do limiar de percolação.

Sun et al. ${ }^{[42]}$ relataram alta adequação do modelo proposto com dados experimentais obtidos para sistemas de NTC/PP, NF/PP e NTC/NF/PP. 
Socher et al. ${ }^{[34]}$ desenvolveram compósitos híbridos de Poliamida 12 com NTC e NF com o objetivo de estudar a condutividade elétrica na mistura. Foram separados em dois grupos de concentrações das cargas, $50 \%$ de NTC com $50 \%$ NF em massa e $75 \%$ de NTC com $25 \%$ de NF em massa. Os autores observaram que os limiares de percolação elétricos obtidos experimentalmente foram maiores do que os calculados pela Equação 21, indicando que o modelo de Sun não se adequou ao sistema e que não houve algum efeito sinergético no limiar de percolação elétrico para estes sistemas híbridos. A explicação foi dada pela formação da rede de percolação estar associada a fatores macroscópicos não descritos na Equação 21, como por exemplo, a viscosidade que é altamente dependente da variação da concentração das cargas individuais dentro da matriz e influencia a condutividade do sistema.

Chen et al. ${ }^{[43]}$ realizaram um dos estudos mais recentes sobre modelagem aplicada ao modelo do volume excluído e baseado no estudo de Sun et al. ${ }^{[42]}$. Os autores realizaram um estudo numérico da percolação elétrica de compósitos poliméricos híbridos de NTC e NF. O limiar de percolação foi estimado pelo método de simulação de Monte Carlo, mirando a avaliação do sinergismo das cargas. Este tópico, já discutido por outros autores ${ }^{[34,42,44,50]}$, foi desenvolvido a partir da Equação 20, porém mais especificamente, com o objetivo de entender melhor este efeito sinérgico e estudar os fatores que afetam o limiar de percolação elétrico destes compósitos híbridos, como a fração volumétrica, razão de aspecto, e diâmetro dos aglomerados de NF. Para isto, a modelagem geométrica da cargas foi fundamental. O NTC foi comumente modelado como o esferocilindro ilustrado na Figura 3. Já o NF possui uma estrutura formada por agregados de partículas nanométricas. Os autores consideraram ser o comprimento dos agregados estatisticamente o mesmo em todas as direções, formando assim uma esfera hipotética com diâmetro igual ao comprimento médio dos agregados. A esfera hipotética não é preenchida apenas com NF, mas também pela matriz polimérica, para a qual a fração em volume de polímero na esfera hipotética foi assumida como $70 \%$. Tanto o modelo do esferocilindro como o das esferas hipotéticas foram distribuídos aleatoriamente pela simulação de Monte Carlo, dentro um volume cúbico representativo, para a montagem da estutura de simulação dos compósitos híbridos. Os autores partiram das considerações clássicas dos modelos já citados, adequaram o modelo de Sun incluindo as constantes $a$ e $b$, que representam as geometrias do esferocilindro e da esfera hipotética, conforme a Equação 22.

$$
\left(1-\frac{V_{N T C}}{\varphi_{c, N T C}}\right)^{a}+\left(1-\frac{V_{N F}}{\varphi_{c, N F}}\right)^{b}=1
$$

Se nenhum efeito sinérgico existir, a relação se torna linear, com os valores de $a$ e $b$ iguais a 1, e a Equação 22 regressa ao modelo Sun et al. ${ }^{[42]}$, Equação 20.

Os autores variaram a razão de aspecto dos NTC e encontraram valores de $a$ e $b$ de 1,478 e 2,179, respectivamente, para uma razão de aspecto de 300 , para o qual foi obtido o menor limiar de percolação, e concluíram que a fração volumétrica de NF necessária para atingir o limiar é muito menor do que o previsto pelo modelo de Sun et al. ${ }^{[42]}$.
Este comportamento não-linear e a menor fração volumétrica de NF indicam o efeito sinérgico, que foi observado também experimentalmente ${ }^{[34,44]}$.

Como conclusão do estudo, os autores mostraram haver uma relação entre os diâmetros das cargas na definição do expoente $b$, como mostrado na Equação 23.

$$
b=0,622+0,0531 \frac{d_{N F}}{d_{N T C}}
$$

Onde $d_{N F}$ e $d_{N T C}$ são, respectivamente, os diâmetros da esfera hipotética e do esferocilindro, revelando que para a determinação da fração volumétrica de percolação elétrica de compósitos híbridos, $\varphi_{C, N T C / N F}$, necessita-se dos valores de $V_{N T C}, V_{N F}, \varphi_{C, N T C}$ e $\varphi_{C, N F}$, bem como da geometria e das dimensões das cargas envolvidas, como descrito na Equação 24.

$\varphi_{c, N T C / N F}=\varphi_{c, N T C}\left\{1-\left[\left(1-\frac{V_{N F}}{\varphi_{c, N F}}\right)^{0,622+0,0531 \frac{d_{N F}}{d_{N T C}}}\right]^{0,690}\right\}+V_{N F}$

Onde $V_{N T C}=\varphi_{C, N T C / N F}-V_{N F}$.

Wu et al. ${ }^{[30]}$ avaliaram o efeito de sinergismo na condutividade elétrica de sistemas com NF e NTC em matriz polimérica biodegradável de poli(ácido láctico) (PLA). O processo de preparação foi realizado em duas etapas. Primeiramente, os compósitos foram preparados em reômetro pela mistura dos materiais no estado fundido; posteriormente, um processo inovador foi realizado, onde a preparação dos compósitos envolveu a formação de uma espuma de PLA sólido com as cargas, pela aplicação de dióxido de carbono em alta pressão na matriz polimérica ${ }^{[45]}$. Para o PLA, a formação de espuma com uma pressão elevada resultou numa estrutura de poros uniformes. $\mathrm{O}$ resultado mostrou-se muito interessante, pois o método de preparo influenciou significantemente no sinergismo na condutividade elétrica. Quando a simples mistura no fundido foi realizada, o efeito sinérgico não foi observado. Porém, quando a formação do compósito em formato espuma foi avaliada, o sinergismo foi pronunciado. Neste estudo a análise do sinergismo na condutividade elétrica foi realizada pela simples avaliação do comportamento aditivo previsto pela Equação 25. Assumindo que as concentrações de NF e NTC de carbono são independentes entre si na matriz de PLA, a condutividade, $\sigma_{N F / N T C}$ pode ser calculada.

$$
\sigma_{N F / N T C}=\varphi_{N F} \sigma_{N F}+\varphi_{N T C} \sigma_{N T C}
$$

Enquanto que os valores experimentais de condutividade elétrica foram inferiores aos calculados pela Equação 25 para os compósitos preparados no estado fundido os compósitos expandidos atingiram valores superiores próximos a 8 ordens de grandeza para concentrações da ordem de $1 \%$ de NF e $1 \%$ de NTC demonstrando a influência da estrutura e do processamento na formação do sistema híbrido ${ }^{[30]}$.

\section{Discussão}

A presente revisão apresentou uma discussão geral dos modelos propostos e aplicados para explicar o comportamento de condutividade elétrica de compósitos poliméricos com cargas 
condutoras. A teoria da percolação é o ponto de partida para explicar o fenômeno e para o desenvolvimento de modelos matemáticos. Os modelos clássicos estatísticos são muito utilizados, porém estão limitados por não considerarem o efeito do tamanho e formato das partículas. Os geométricos, embora assumam estas variáveis, descrevem adequadamente os sistemas submetidos ao processo de sinterização e não são aplicáveis aos principais métodos de preparação dos compósitos poliméricos. O modelo de volume excluído, por sua vez, apresenta a possibilidade da inserção destas variáveis geométricas como forma, tamanho e razão de aspecto, e tem se mostrado o de maior potencial na predição do limiar de percolação em sistemas de polímeros modificados com cargas condutoras.

Como são possíveis interações entre cargas com geometrias variadas ou mesmo de diferentes naturezas, como as carbonáceas e metálicas, modelos com base em dados empíricos devem ser adotados com cuidado, reforçando a necessidade de maior investigação do comportamento destes sistemas.

\section{Referências}

1. Hori, M., Aoki, T., Ohira, Y., Yano, S., Hori, M., Aoki, T., Ohira, Y., \& Yano, S. (2001). New type of mechanical damping composites composed of piezoelectric ceramics, carbon Black and epoxy resin. Composites. Part A, Applied Science and Manufacturing, 32(2), 287-290. http://dx.doi.org/10.1016/ S1359-835X(00)00141-X.

2. Marcq, F., Demont, P., Monfraix, P., Peigney, A., Laurent, C., Falat, T., Courtade, F., \& Jamin, T. (2011). Carbon nanotubes and silver flakes filled epoxy resin for new hybrid conductive adhesives. Microelectronics and Reliability, 51(7), 1230-1234. http://dx.doi.org/10.1016/j.microre1.2011.03.020.

3. Li, C., Liang, T., Lu, W., Tang, C., Hu, X., Cao, M., \& Liang, J. (2004). Improving the antistatic ability of polypropylene fibers by inner antistatic agent filled with carbon nanotubes. Composites Science and Technology, 64(13-14), 2089-2096. http://dx.doi.org/10.1016/j.compscitech.2004.03.010.

4. Chung, D. D. L. (2001). Review: electromagnetic interference shielding effectiveness of carbon materials. Carbon, 39(2), 279-285. http://dx.doi.org/10.1016/S0008-6223(00)00184-6.

5. Ryan, M. A., Shevade, A. V., Zhou, H., \& Homer, M. L. (2004). Polymer-carbon black composite sensors in an Electronic Nose for air-quality monitoring. MRS Bulletin, 29(10), 714-719. PMid:15991400. http://dx.doi.org/10.1557/mrs2004.208.

6. Al-Saleh, M. H., \& Sundararaj, U. (2008). Nanostructured carbon black filled polypropylene/polystyrene blends containing styrene-butadiene-styrene copolymer: Influence of morphology on electrical resistivity. European Polymer Journal, 44(7), 1931-1939. http://dx.doi.org/10.1016/j.eurpolymj.2008.04.013.

7. Ajayan, P. M. (1999). Nanotubes from carbon. Chemical Reviews, 99(7), 1787-1800. PMid:11849010. http://dx.doi. org/10.1021/cr970102g.

8. Mamunya, Y. P., Zois, H., Apekis, L., \& Lebedev, E. V. (2004). Influence of pressure on the electrical conductivity of metal powders used as fillers in polymer composites. Powder Technology, 140(1-2), 49-55. http://dx.doi.org/10.1016/j. powtec.2003.11.010.

9. Sonoda, K., Teirikangas, M., Juuti, J., Moriya, Y., \& Jantunen, H. (2011). Effect of surface modification on dielectric and magnetic properties of metal powder/polymer nanocomposites. Journal of Magnetism and Magnetic Materials, 323(17), 22812286. http://dx.doi.org/10.1016/j.jmmm.2011.04.007.
10. Chen, G. H., Wu, D. J., Weng, W. G., \& Yan, W. L. (2001). Preparation of polymer/graphite conducting nanocomposites by intercalation polymerization. Journal of Applied Polymer Science, 82(10), 2506-2513. http://dx.doi.org/10.1002/app.2101.

11. Zou, J. F., Yu, Z. Z., Pan, Y. X., Fang, X. P., \& Ou, Y. C. (2002). Conductive mechanism of polymer/graphite conducting composites with low percolation threshold. Journal of Polymer Science. Part B, Polymer Physics, 40(10), 954-963. http:// dx.doi.org/10.1002/polb.10141.

12. Zheng, W., \& Wong, S. C. (2003). Electrical conductivity and dielectric properties of PMMA/expanded graphite composites. Composites Science and Technology, 63(2), 225-236. http:// dx.doi.org/10.1016/S0266-3538(02)00201-4.

13. Sun, Y., Luo, S., Watkins, K., \& Wong, C. P. (2004). Electrical approach to monitor the thermal oxidation aging of carbon black filled ethylene propylene rubber. Polymer Degradation \& Stability, 86(2), 209-215. http://dx.doi.org/10.1016/j. polymdegradstab.2004.04.013.

14. Ramasubramaniam, R., Chen, J., \& Liu, H. (2003). Homogeneus carbon nanotubes/polymer composites for electrical applications. Applied Physics Letters, 83(14), 2928-2930. http://dx.doi. org/10.1063/1.1616976.

15. Biercuk, M. J., Llaguno, M. C., Radosavljevic, M., Hyun, J. K., Johnson, A. T., \& Fischer, J. E. (2002). Carbon nanotube composites for thermal management. Applied Physics Letters, 80(15), 15-23. http://dx.doi.org/10.1063/1.1469696.

16. Weisenberger, M. C., Grulke, E. A., Jacques, D., Rantell, T. \& Andrews, R. (2003). Enhanced mechanical properties of polyacrylonitrile/multiwall carbon nanotube camposite fibers. Journal of Nanoscience and Nanotechnology, 3(6), 535-539. http://dx.doi.org/10.1166/2003.239.

17. Spitalsky, Z., Tasis, D., Papagelis, K., \& Galiotis, C. (2010). Carbon nanotube-polymer composites: chemistry, processing, mechanical and electrical properties. Progress in Polymer Science, 35(3), 357-401. http://dx.doi.org/10.1016/j. progpolymsci.2009.09.003.

18. Ma, P.-C., Siddiqui, N. A., Marom, G. J., \& Kim, K. (2010). Dispersion and functionalization of carbon nanotubes for polymer-based nanocomposites: a review. Composites. Part A, Applied Science and Manufacturing, 41(10), 1345-1367. http://dx.doi.org/10.1016/j.compositesa.2010.07.003.

19. Lux, F. (1993). Models proposed to explain the electrical conductivity of mixtures made of conductive and insulating materials. Journal of Materials Science, 28(2), 285-301. http:// dx.doi.org/10.1007/BF00357799.

20. Bauhofer, W., \& Kovacs, J. Z. (2009). A review and analysis of electrical percolation in carbon nanotube polymer composites. Composites Science and Technology, 69(10), 1486-1498. http:// dx.doi.org/10.1016/j.compscitech.2008.06.018.

21. György, I. (2008). Conducting polymers a new era in electrochemistry. Berlin Heidelberg: Springer-Verlag.

22. Hoppe, H., \& Sariciftci, N. S. (2004). Organic solar cells: an overview. Journal of Materials Research, 19(7), 1924-1945. http://dx.doi.org/10.1557/JMR.2004.0252.

23. Winey, K. I., Kashiwagi, T., \& Mu, M. (2007). Improving electrical conductivity and thermal properties of polymers by the addition of carbon nanotubes as fillers. MRS Bulletin, 32(4), 348-353. http://dx.doi.org/10.1557/mrs2007.234.

24. Ounaies, Z., Park, C., Wise, K. E., Siochi, E. J., \& Harrison, J. S. (2003). Electrical properties of single wall carbon nanotube reinforced polyimide composites. Composites Science and Technology, 63(11), 1637-1646. http://dx.doi.org/10.1016/ S0266-3538(03)00067-8.

25. Iijima, S. (1991). Helical microtubules of graphitic carbon. Nature, 356(6348), 56-54. http://dx.doi.org/10.1038/354056a0. 
26. Balberg, I., Anderson, C. H., Alexander, S., \& Wagner, N. (1984). Excluded volume and its relation to the onset of percolation. Physical Review B: Condensed Matter and Materials Physics, 30(7), 3933-3943. http://dx.doi.org/10.1103/PhysRevB.30.3933.

27. Huang, J. C. (2002). Carbon black filled conducting polymers and polymer blends. Advances in Polymer Technology, 21(4), 299-313. http://dx.doi.org/10.1002/adv.10025.

28. Ou, R., Gupta, S., Parker, C. A., \& Gerhardt, R. A. (2006). Fabrication and electrical conductivity of poly(methyl methacrylate) (PMMA)/carbon black (CB) composites: comparison between an ordered carbon black nanowire-like segregated structure and a randomly dispersed carbon black nanostructure. The Journal of Physical Chemistry B, 110(45), 22365-22373. PMid:17091976. http://dx.doi.org/10.1021/jp064498o.

29. Scherzer, S. L., Pavlova, E., Esper, J. D., \& Starý, Z. (2015). Phase structure, rheology and electrical conductivity of cocontinuous polystyrene/polymethylmethacrylate blends filled with carbon black. Composites Science and Technology, 1-29. http://dx.doi.org/10.1016/j.compscitech.2015.10.003.

30. Wu, D., Lv, Q., Feng, S., Chen, J., Chen, Y., Qiu, Y., \& Yao, X. (2015). Polylactide composite foams containing carbon nanotubes and carbon black: Synergistic effect of filler on electrical conductivity. Carbon, 95, 380-387. http://dx.doi. org/10.1016/j.carbon.2015.08.062.

31. Pelíšková, M., Piyamanocha, P., Prokeš, J., Varga, M., \& Sáha, P. (2014). The electrical conductivity of ethylene butylacrylate/carbon black composites: The effect of foaming on the percolation threshold. Synthetic Metals, 188, 140-145. http://dx.doi.org/10.1016/j.synthmet.2013.12.008.

32. Ebbesen, T. W., Lezec, H. J., Hiura, H., Bennett, J. W., Ghaemi, H. F., \& Thio, T. (1996). Electrical conductivity of individual carbon nanotubes. Nature, 382(6586), 54-56. http://dx.doi. org/10.1038/382054a0.

33. Sandler, J. K. W., Kirk, J. E., Kinloch, I. A., Shaffer, M. S. P., \& Windle, A. H. (2003). Ultra-low electrical percolation threshold in carbon-nanotube-epoxy composites. Polymer, 44(19), 58935899. http://dx.doi.org/10.1016/S0032-3861(03)00539-1.

34. Socher, R., Krause, B., Hermasch, S., Wursche, R., \& Pötschke, P. (2011). Electrical and thermal properties of polyamide 12 composites with hybrid fillers systems of multiwalled carbon nanotubes and carbon black. Composites Science and Technology, 71(8), 1053-1059. http://dx.doi.org/10.1016/j. compscitech.2011.03.004.

35. Park, S. J., Lim, S. T., Cho, M. S., Kim, H. M., Joo, J., \& Choi, H. J. (2005). Electrical properties of multi-walled carbon nanotube/poly(methyl methacrylate) nanocomposite. Current Applied Physics, 5(4), 302-304. http://dx.doi.org/10.1016/j. cap.2004.02.013.

36. Zhao, J., Dai, K., Liu, C., Zheng, G., Wang, B., Liu, C., Chen, J. \& Shen, C. (2013). A comparation between strain sensing behaviors of carbon black/polypropylene and carbon nanotubes/ polypropylene electrically conductive composites. Composites Part A: Applied Science and Manucfacturing, 48, 129-136. http://dx.doi.org/10.1016/j.compositesa.2013.01.004.

37. Chen, G. H., Wu, C. L., Weng, W. G., Wu, D. J., \& Yan, W. L. (2003). Preparation of polystyrene/graphite nanosheet composite. Polymer, 44(6), 1781-1784. http://dx.doi.org/10.1016/S00323861(03)00050-8.

38. Weng, W. G., Chen, G. H., \& Wu, D. J. (2005). Transport properties of electrically conducting nylon 6/foliated graphite nanocomposites. Polymer, 46(16), 6250-6257. http://dx.doi. org/10.1016/j.polymer.2005.05.071.

39. Kamat, P. V., Thomas, K. G., Barazzouk, S., Girishkumar, G., Vinodgopal, K., \& Meisel, D. (2004). Self-assembled linear bundles of single wall carbon nanotubes and their alignment and deposition as a film in a dc field. Journal of the American
Chemical Society, 126(34), 10757-10762. PMid:15327336. http://dx.doi.org/10.1021/ja0479888.

40. Chen, Z., Yang, Y. L., Wu, Z. Y., Luo, G., Xie, L. M., Liu, Z. F., Ma, S., \& Guo, W. (2005). Electric-field-enhanced assembly of single-walled carbon nanotubes on a solid surface. The Journal of Physical Chemistry B, 109(12), 5473-5477. PMid:16851584. http://dx.doi.org/10.1021/jp045796t.

41. Takahashi, T., Murayama, T., Higuchi, A., Awano, H., \& Yonetake, K. (2006). Aligning vapor-grown carbon fibers in polydimethylsiloxane using dc electric or magnetic field. Carbon, 44(7), 1180-1188. http://dx.doi.org/10.1016/j. carbon.2005.10.055.

42. Sun, Y., Bao, H.-D., Guo, Z.-X., \& Yu, J. (2009). Modeling of the electrical percolation of mixed carbon fillers in polymerbased composites. Macromolecules, 42(1), 459-463. http:// dx.doi.org/10.1021/ma8023188.

43. Chen, Y., Wang, S. Pan, F. \& Zhang, J. (2014). A numerical study on electrical percolation of polymer-matrix composites with hybrid fillers of carbon nanotubes and carbon black. Journal of Nanomaterials, 2014, 1-9. http://dx.doi.org/10.1155/2014/614797.

44. Wen, M., Sun, X., Su, L., Shen, J., Li, J., \& Guo, S. (2012). The electrical conductivity of carbon nanotube/carbon black/ polypropylene composites prepared through multistage stretching extrusion. Polymer, 53(7), 1602-1610. http://dx.doi. org/10.1016/j.polymer.2012.02.003.

45. Nofar, M., \& Park, C. B. (2014). Poly(lactic acid) foaming. Progress in Polymer Science, 39(10), 1721-1741. http://dx.doi. org/10.1016/j.progpolymsci.2014.04.001.

46. Clingerman, L. M., Weber, E. H., King, J. A., \& Schulz, K. H. (2002). Synergistic effect of fillers in electrically conductive nylon 6,6 and polycarbonate based resins. Polymer Composites, 23(5), 911-924. http://dx.doi.org/10.1002/pc.10488.

47. Thongruang, W., Spontak, R. J., \& Balik, C. M. (2002). Correlated electrical conductivity and mechanical property analysis of high-density polyethylene filled with graphite and carbon fiber. Polymer, 43(8), 2279-2286. http://dx.doi. org/10.1016/S0032-3861(02)00043-5.

48. Krause, B., Pötschke, P., \& Häußler, L. (2009). Influence of small scale melt mixing conditions on electrical resistivity of carbon nanotube-polyamide composites. Composites Science and Technology, 69(10), 1505-1515. http://dx.doi.org/10.1016/j. compscitech.2008.07.007.

49. Kasaliwal, G., Göldel, A., \& Pötschke, P. (2009). Influence of processing conditions in smallscale melt mixing and compression molding on the resistivity and morphology of polycarbonateMWNT composites. Journal of Applied Polymer Science, 112(6), 3494-3509. http://dx.doi.org/10.1002/app.29930.

50. Sumfleth, J., Adroher, X. C., \& Schulte, K. (2009). Synergistic effects in network formation and electrical properties of hybrid epoxy nanocomposites containing multi-wall carbon nanotubes and carbon black. Journal of Materials Science, 44(12), 32413247. http://dx.doi.org/10.1007/s10853-009-3434-7.

51. Bokobza, L., Rahmani, M., Belin, C., Bruneel, J. L., \& El Bounia, N. E. (2008). Blends of carbon blacks and multiwall carbon nanotubes as reinforcing fillers for hydrocarbon rubbers. Journal of Polymer Science. Part B, Polymer Physics, 46(18), 1939-1951. http://dx.doi.org/10.1002/polb.21529.

52. Calleja, F. J. B., Bayer, R. K., \& Ezquerra, T. A. (1988). Electrical conductivity of polyethylene-carbon-fibre composites mixed with carbon black. Journal of Materials Science, 23(4), 1411-1415. http://dx.doi.org/10.1007/BF01154609.

53. Lee, J. H., Kim, S. K., \& Kim, N. H. (2006). Effects of the addition of multi-walled carbon nanotubes on the positive temperature coefficient characteristics of carbonblackfilled high-density polyethylene nanocomposites. Scripta 
Materialia, 55(12), 1119-1122. http://dx.doi.org/10.1016/j. scriptamat.2006.08.051.

54. Bhattacharya, S. K., editor (1986). Metal-filled polymers (properties and applications). New York: Marcel Dekker.

55. Chen, I.-G., \& Johnson, W. B. (1991). Alternating-current electrical properties of random metal-insulator composites. Journal of Materials Science, 26(6), 1565-1576. http://dx.doi. org/10.1007/BF00544665.

56. Carmona, F., \& Mouney, C. (1992). Temperature-dependent resistivity and conduction mechanism in carbon particle-filled polymers. Journal of Materials Science, 27(5), 1322-1326. http://dx.doi.org/10.1007/BF01142046.

57. Boiteux, G., Fournier, J., Issotier, D., Scytre, G., \& Marichy, G. (1999). Conductive thermoset composites: PTC effect. Synthetic Metals, 102(1-3), 1234-1235. http://dx.doi.org/10.1016/ S0379-6779(98)01432-5.

58. Coleman, J. N., Khan, U., Blau, W. J., \& Gun'ko, Y. K. (2006). Small but strong: a review of the mechanical properties of carbon nanotube-polymer composites. Carbon, 44(9), 16241652. http://dx.doi.org/10.1016/j.carbon.2006.02.038.

59. Moniruzzaman, M., \& Winey, K. I. (2006). Polymer nanocomposites containing carbon nanotubes. Macromolecules, 39(16), 5194-5205. http://dx.doi.org/10.1021/ma060733p.

60. Breuer, O., \& Sundararaj, U. (2004). Big returns from small fibers: a review of polymer/carbon nanotube composites. Polymer Composites, 25(6), 630-645. http://dx.doi.org/10.1002/ pc.20058.

61. Sahoo, N. G., Rana, S., Cho, J. W., Li, L., \& Chan, S. H. (2010). Polymer nanocomposites based on functionalized carbon nanotubes. Progress in Polymer Science, 35(3), 837867. http://dx.doi.org/10.1016/j.progpolymsci.2010.03.002.

62. Strümpler, R., \& Glatz-Reichenbach, J. (1999). Conducting polymers composites. Journal of Electroceramics, 3(4), 329346. http://dx.doi.org/10.1023/A:1009909812823.

63. Broadbent, S. R., \& Hammersley, J. M. (1957). Percolation processes: I. Crystals and mazes. Mathematical Proceedings of the Cambridge Philosophical, 53(3), 629-641. http://dx.doi. org/10.1017/S0305004100032680.

64. Bruggeman, D. A. G. (1935). Berechnung verschiedener physikalischer Konstanten von heterogenen Substanzen. I. Dielektrizitätskonstanten und Leitfähigkeiten der Mischkörper aus isotropen Substanzen. Annalen der Physik, 416(7), 636664. http://dx.doi.org/10.1002/andp.19354160705.

65. Garland, J. C., \& Tanner, D. B., editores (1978). Electrical transport and optical properties of inhomogeneous media. New York: AIP.

66. Xue, Q. Z. (2000). Effective-medium theory for two-pases random composites with an interfacial shell. Journal of Materials Science \& Technology, 16(4), 367-369. Recuperado em 20 de setembro de 2012, de http://www.jourlib.org/paper/1532329\#. VW23gs_BzGc

67. Xue, Q. Z. (2003). A percolation model of metal-insulator composites. Physica B, Condensed Matter, 325(1-4), 195-198. http://dx.doi.org/10.1016/S0921-4526(02)01523-5.

68. Xue, Q. Z. (2004). The influence of particle shape and size on electric conductivity of metal-polymer composites. European Polymer Journal, 40(2), 323-327. http://dx.doi.org/10.1016/j. eurpolymj.2003.10.011.

69. Torquato, S., \& Hyun, S. (2001). Effective-medium theory for composite media: realizable single-scale dispersions. Journal of Applied Physics, 89(3), 1725-1729. http://dx.doi. org/10.1063/1.1336523.

70. Kirkpatrick, S. (1973). Percolation and conduction. Reviews of Modern Physics, 45(4), 574-588. http://dx.doi.org/10.1103/ RevModPhys.45.574.
71. Zallen, R. A. (1983). The physics of amorphous solids. New York: Wiley.

72. Bao, S. P., Liang, G. D., \& Tjong, S. C. (2011). Effect of mechanical stretching on electrical conductivity and positive temperature coefficient characteristics of poly(vinylidene fluoride)/carbon nanofiber composites prepared by nonsolvent precipitation. Carbon, 49(5), 1758-1768. http://dx.doi. org/10.1016/j.carbon.2010.12.062.

73. Pike, G. E., \& Seager, C. H. (1974). Percolation and conductivity: a computer study. I. Physical Review B: Condensed Matter and Materials Physics, 10(4), 1435-1444. http://dx.doi.org/10.1103/ PhysRevB.10.1421.

74. Grunlan, J. C., Mehrabi, A. R., Bannon, M. V., \& Bahr, J. L. (2004). Water-based single-walled-nanotube-filled polymer composite with an exceptionally low percolation threshold. Advanced Materials, 16(2), 150-153. http://dx.doi.org/10.1002/ adma.200305409.

75. Ehrburgerdolle, F., Lahaye, J., \& Misono, S. (1994). Percolation in carbon black powders. Carbon, 32(7), 1363-1368. http:// dx.doi.org/10.1016/0008-6223(94)90123-6.

76. Etemad, S., Quan, X., \& Sanders, N. A. (1986). Geometrydefined electrical interconnection by a homogeneous medium. Applied Physics Letters, 48(9), 607-609. http://dx.doi. org/10.1063/1.96482.

77. Janzen, J. (1975). On the critical conductive filler loading in antistatic composites. Journal of Applied Physics, 46(3), 966-969. http://dx.doi.org/10.1063/1.321629.

78. Slupkowski, T. (1984). Electrical conductivity of mixtures of conducting and insulating particles. Physica Status Solidi, 83(1), 329-333. http://dx.doi.org/10.1002/pssa.2210830137.

79. Scarisbrick, R. (1973). Electrically conducting mixtures. Journal of Physics. D, Applied Physics, 6(17), 2098-2110. http://dx.doi.org/10.1088/0022-3727/6/17/316.

80. Malliaris, A., \& Turner, D. T. (1971). 42 Influence of particle size on the electrical resistivity of compacted mixtures of polymeric and metallic powders. Journal of Applied Physics, 614-618. http://dx.doi.org/10.1063/1.1660071.

81. Youngs, I. J. (2003). A geometric percolation model for nonspherical excluded volumes. Journal of Physics. D, Applied Physics, 36(26), 738-747. http://dx.doi.org/10.1088/00223727/36/6/317

82. Balberg, I. (1987). Recent developments in continuum percolation. Philosophical Magazine B, 56(6), 991-1003. http://dx.doi.org/10.1080/13642818708215336.

83. Bug, A. L. R., Safran, S. A., \& Webman, I. (1985). Continuum percolation of rods. Physical Review Letters, 54(13), 1412-1415. PMid:10031025. http://dx.doi.org/10.1103/PhysRevLett.54.1412.

84. Celzard, A., Mcrae, E., Deleuze, C., Dufort, M., Furdin, G., \& Marêché, J. F. (1996). Critical concentration in percolating systems containing a high-aspect-ratio filler. Physical Review B: Condensed Matter and Materials Physics, 53(10), 6209-6214. PMid:9982020. http://dx.doi.org/10.1103/PhysRevB.53.6209.

85. Wu, S.-H., Masaharu, I., Natsuki, T., \& Ni, Q.-Q. (2006). Electrical conduction and percolation behavior of carbon nanotubes/UPR nanocomposites. Journal of Reinforced Plastics and Composites, 25(18), 1957-1966. http://dx.doi. org/10.1177/0731684406069923.

86. Berhan, L., \& Sastry, A. M. (2007). Modeling percolation in high-aspect-ratio fiber systems. I. Soft-core versus hardcore models. Physical Review E: Statistical, Nonlinear, and Soft Matter Physics, 75(4), 0411201-0411208. http://dx.doi. org/10.1103/PhyRevLett.75.041120. PMid:17500878.

87. Deng, H., Zhang, R., Bilotti, E., Loos, J., \& Peijs, T. (2009). Conductive polymer tape containing highly oriented carbon nanofillers. Journal of Applied Polymer Science, 113(2), 742751. http://dx.doi.org/10.1002/app.29624. 
88. Dalmas, F., Dendievel, R., Chazeau, L., Cavaillé, J.-Y., \& Gauthier, C. (2006). Carbon nanotube-filled polymer composites. Numerical simulation of electrical conductivity in three-dimensional entangled fibrous networks. Acta Materialia, 54(11), 2923-2931. http://dx.doi.org/10.1016/j. actamat.2006.02.028.

89. Berhan, L., \& Sastry, A. M. (2007). Modeling percolation in high-aspect-ratio fiber systems. II. The effect of waviness on the percolation onset. Physical Review E: Statistical, Nonlinear, and Soft Matter Physics, 75(4), 0411211-0411218. http://dx.doi. org/10.1103/PhyRevLett.75.041121. PMid:17500879.

90. Sun, Y.; Bao, H-D.; Guo, Z.-X. \& Yu, J. (2009). Modeling of the electrical percolation of mixed carbon fillers in polymerbased composites. Macromolecules, 42(1), 459-463.

Enviado: Jul. 03, 2015 Revisado: Nov. 07, 2015 Aceito: Dez. 16, 2015 